\title{
PERFIL DE ESTEROIDES SEXUALES DEL CAPITÁN DE LA SABANA (Eremophilus mutisii) DURANTE UN CICLO HIDROLOGICO COMPLETO
}

\section{PROFILE OF SEXUAL STEROIDS OF THE CAPITÁN DE LA SABANA (Eremophilus mutisii) DURING A COMPLETE HYDROLOGICAL CYCLE}

\author{
Miguel A. Landines P. ${ }^{1}$, Camilo A. Prieto M. $^{2}$, Liliana Rodríguez V. ${ }^{3}$, Rafael Rosado P. ${ }^{4}$
}

\begin{abstract}
${ }^{1}$ Zootecnista, Ph.D., Laboratorio de Fisiología de Peces, Facultad de Medicina Veterinaria y de Zootecnia. Universidad Nacional de Colombia, e-mail: malandinezp@unal.edu.co; ${ }^{2}$ Zootecnista, M.Sc., Ph.D., Profesor, Facultad de Ciencias Pecuarias, Programa de Zootecnia. Universidad de Ciencias Aplicadas y Ambientales U.D.C.A, Bogotá, Colombia, e-mail: camprieto@ udca.edu.co; ${ }^{3}$ Zootecnista, M.Sc., Laboratorio de Fisiología de Peces, Facultad de Medicina Veterinaria y de Zootecnia. Universidad Nacional de Colombia, e-mail: Irodriguezv@unal.edu.co; ${ }^{4}$ Biólogo Marino, M.Sc., Laboratorio de Fisiología de Peces, Facultad de Medicina Veterinaria y de Zootecnia. Universidad Nacional de Colombia, e-mail: rrosadop@unal.edu.co. Carrea 30 No. 45-03, Ciudad Universitaria, Edificio 481, Laboratorio 4. Bogotá-Colombia.
\end{abstract}

Rev. U.D.C.A Act. \& Div. Cient. 20(1): 43-50, Enero Junio, 2017

\section{RESUMEN}

Fueron capturados, mensualmente, adultos de Eremophilus mutisii, en la parte alta del río Bogotá (Municipio de Suesca). Los ejemplares fueron anestesiados, pesados y medidos. Posteriormente, se tomaron muestras de sangre, se extrajeron y se pesaron las gónadas, para cálculo del índice gonadosomático (IGS). Se determinaron testosterona (T) y estradiol (E2), por quimioluminiscencia. La proporción de hembras fue mayor que la de machos, existiendo muestreos en los que solo se obtuvieron hembras. Los niveles de $\mathrm{T}$ en hembras permanecieron muy bajos durante todo el ciclo, manteniéndose así o indetectables, con un único pico en octubre. En machos, los niveles fueron superiores, alcanzando valores mayores en abril, septiembre, noviembre y diciembre, con pico en noviembre. El E2 en hembras mostró niveles variables, observándose niveles elevados durante noviembre, diciembre y enero, con pico en diciembre. En machos, solo fue posible determinar E2 en noviembre, diciembre, marzo, abril y mayo, puesto que durante febrero, junio, agosto y septiembre, los valores fueron indetectabes y en los meses restantes, no se capturó ningún macho. El valor máximo fue en noviembre y el mínimo en marzo y abril. En hembras, los picos de E2 coincidieron con los valores más altos de IGS, mientras que la T mantuvo su valor bajo, independientemente de dicho índice. En machos, la relación entre T e IGS también fue coincidente, aunque no de manera tan evidente como en hembras. No se observó una tendencia clara entre la relación de E2 e IGS para machos.
Palabras clave: Testosterona, Estradiol, IGS, Reproducción de peces.

\section{SUMMARY}

Specimens of Eremophilus mutisii were captured monthly on the upper part of the Bogotá River (municipality of Suesca). The specimens were anesthetized, weighed and measured. Subsequently, a blood sample was taken. The gonads were extracted and weighed to calculate the gonadosomatic index (GSI). Testosterone (T) and estradiol (E2) were determined by chemiluminescence technique. The proportion of females was always greater than that of males, and there were months in which only females were obtained. The $T$ values of the females remained very low during the whole cycle, remaining low or imperceptible levels, with a single peak in October. In males, levels were always higher reaching high values in April, September, November, and December, with a peak in November. E2 in females showed variables values with high levels in November, December and January with a peak in December. In males, it was only possible to determine E2 in November, December, March, April and May, since, during February, June, August, and September the values were imperceptible and in the remaining months no male was captured. The maximum value was obtained in November and the minimum in March and April. In females, the peaks of E2 coincided with the higher values of GSI, while T maintained its low value, independently of this index. In males, the relationship between T and GSI was 
also coincident, although not as evident as in females. Also, a clear trend was not observed between the E2 and GIS ratio for males.

Key words: Testosterone, Estradiol, GSI, Fish reproduction.

\section{INTRODUCCIÓN}

El capitán de la sabana, Eremophilus mutisii Humboldt (Siluriformes: Trichomyteridae) es la especie íctica representativa y emblemática del río Bogotá y sus afluentes y desde tiempos ancestrales ha sido importante en la región del altiplano cundiboyacense (Maldonado et al. 2008), formando parte habitual de la dieta de los pobladores de la región, incluso, desde tiempos pasados, cuando comunidades indígenas nativas habitaban la zona; sin embargo, el actual deterioro de los ecosistemas, fundamentalmente por acción antrópica, ha diezmado sus poblaciones (Salcedo et al. 2012), al punto de pasar en la clasificación de la UICN de categoría "Casi Amenazada", en el 2002 (Mojica et al. 2002), a "Vulnerable", en el 2012 (Mojica et al. 2012). Este hecho exige un trabajo de conservación de la especie y de su hábitat que, eventualmente, permita su recuperación. Por otro lado, ha sido catalogada por varios autores como, probablemente, la única especie nativa de aguas frías, con posibilidad de ser utilizada para piscicultura (Rosado \& González, 2007). Esta actividad, en la actualidad, se realiza de manera exclusiva, con una especie introducida, la trucha arcoíris.

Con base en lo anterior, en diversos escenarios, se ha reiterado la importancia de realizar investigaciones con la especie, tendientes a evitar su probable desaparición y promover su posible incorporación a los sistemas de producción piscícola en cautiverio (Valderrama et al. 2011). Para ello, el punto de partida es el conocimiento biológico de la especie, el cual, si bien es cierto ya ha sido abordado por varios autores (González \& Rosado, 2005), quienes han adelantado estudios sobre biología básica, ecología, alimentación, patología y hasta reproducción artificial y cultivo experimental (Rodríguez \& Rosado, 1993), aún no se registra información suficiente sobre su biología básica y reproductiva y no se ha explorado la viabilidad de ser introducida a los sistemas de cultivo.

De hecho, el volumen de información disponible sobre la especie es limitado, tal como se evidencia en las revisiones que, sobre el particular, realizaron Rosado \& González (2007) y González \& Rosado (2010), quienes resaltan que la mayoría de las investigaciones realizadas con la especie son valoraciones biológicas y ecológicas; sin embargo, como se mencionó, aún no se conoce, a ciencia cierta, toda la biología reproductiva de la especie en el medio natural y, mucho menos, el perfil de hormonas que regulan sus ciclos re- productivos, parámetros de trascendental importancia para entender la reproducción en la naturaleza, con miras a una eventual replicación en cautiverio. En tal sentido, el propósito del presente trabajo fue obtener el perfil de esteroides sexuales de adultos de la especie en condiciones naturales, durante un ciclo hidrológico completo, el cual, junto con la descripción del desarrollo gonadal, son los parámetros más importantes para el entendimiento de su biología y fisiología reproductivas.

\section{MATERIALES Y MÉTODOS}

Localización. El área de colecta fue la parte alta del río Bogotá, en inmediaciones de la provincia de Almeidas, localizada al nororiente del departamento de Cundinamarca, a la altura del municipio de Suesca, a $05^{\circ} 06^{\prime}$ latitud Norte y $73^{\circ} 48^{\prime}$ longitud Oeste y a $2584 \mathrm{msnm}$. El análisis del material biológico y la determinación de los esteroides sexuales, se llevaron a cabo en el Laboratorio de Fisiología de Peces, adscrito a la Facultad de Medicina Veterinaria y de Zootecnia, de la Universidad Nacional de Colombia, sede Bogotá.

Material biológico. Fueron utilizados 83 individuos adultos de capitán de la Sabana, obtenidos de pesquerías mensuales durante un año, los cuales, en cada una de las fechas de pesca, fueron anestesiados, pesados y medidos in situ. Posteriormente, por punción en la vena caudal, se tomó una muestra de sangre de cada individuo, la cual, fue centrifugada durante 7 minutos, a $2.500 \mathrm{~g}$, para la obtención del plasma, que fue transportado, debidamente refrigerado, hasta el laboratorio, para su posterior análisis.

Adicionalmente, fueron extraídas las gónadas de todos los ejemplares para su pesaje, en balanza digital, con aproximación a 0,01g, para determinar el índice gonadosomático (IGS), según la siguiente expresión matemática:

$$
\text { IGS }=(\text { Peso gónada/Peso total }) * 100
$$

Determinación de esteroides. En el laboratorio, se determinaron testosterona (T) y estradiol (E2), a través de la técnica de Quimioluminiscencia (CLIA), utilizando kits comerciales Acculite CLIA Microwells (Monobind Inc. ${ }^{\circledR)}$ ): Testosterone Kit System 3775-300 y Estradiol Kit System 4975-300, respectivamente.

La realización de las pruebas, incluyendo el montaje, la incubación, el lavado y la lectura, se llevó a cabo según el manual de procedimientos del Laboratorio de Fisiología de Peces de la Universidad Nacional de Colombia. La lectura final, se llevó a cabo en el equipo LumiStat 4100 de Awareness Technology ${ }^{\circledR}$. 


\section{RESULTADOS Y DISCUSIÓN}

En total, se capturaron 83 animales, de los cuales, 56 fueron hembras y 27 machos, obteniéndose una relación hembra/ macho de 2,03/1. En la tabla 1, se presentan los valores medios de peso e IGS obtenidos, tanto para hembras como para machos, en todos los meses, pudiéndose observar que, en junio, no fue posible capturar individuos y, en varios de los meses restantes, solamente fueron capturadas hembras, por tal motivo, no fue posible tener información de junio.

En la figura 1, se presentan los valores de testosterona encontrados a lo largo del año, tanto para hembras como para machos, pudiéndose observar que, en las hembras, los niveles permanecieron muy bajos durante todo el ciclo, manteniéndose en valores indetectables o cercanos a $0,01 \mathrm{ng} / \mathrm{mL}$, con un único pico de $0,03 \mathrm{ng} / \mathrm{mL}$ en octubre. En los machos, los niveles fueron superiores a los de las hembras, alcanzando valores mayores a $0,1 \mathrm{ng} / \mathrm{mL}$ en abril, septiembre, noviembre y diciembre, con pico en noviembre $(0,19 \mathrm{ng} / \mathrm{mL})$.

Tales resultados, son similares a los encontrados por Sisneros et al. (2004), en Porichthys notatus y por Shabana et al. (2013), en Argyrosomus regius, especies, en las que se observaron, en los machos, fluctuaciones en los niveles de testosterona, con aumento significativo en las épocas de maduración, con un posterior descenso en animales ya maduros; sin embargo, tal descenso ocurriría después del desove, tal como lo reportan Schiavone et al. (2012), quienes encontraron niveles aumentados de esteroides durante la época de desove de Argyrosomus regio.

Tabla 1. Valores medios de peso e Índice Gonadosomático (IGS) de Eremophilus mutisii, capturados en el municipio de Suesca (Cundinamarca).

\begin{tabular}{|c|c|c|c|c|}
\hline Mes & Sexo & $\mathbf{n}$ & Promedio de Peso (g) & IGS (\%) \\
\hline \multirow{2}{*}{ Octubre } & Hembra & 9 & 92,84 & 11,93 \\
\hline & Macho & 0 & SD & SD \\
\hline \multirow{2}{*}{ Noviembre } & Hembra & 2 & 91,45 & 13,31 \\
\hline & Macho & 2 & 101,80 & 7,56 \\
\hline \multirow{2}{*}{ Diciembre } & Hembra & 4 & 101,33 & 17,05 \\
\hline & Macho & 3 & 113,87 & 4,41 \\
\hline \multirow{2}{*}{ Enero } & Hembra & 6 & 82,43 & 15,02 \\
\hline & Macho & 0 & SD & SD \\
\hline \multirow{2}{*}{ Febrero } & Hembra & 2 & 82,15 & 5,10 \\
\hline & Macho & 3 & 52,73 & 3,13 \\
\hline \multirow{2}{*}{ Marzo } & Hembra & 1 & 159,00 & 21,07 \\
\hline & Macho & 2 & 119,40 & 4,34 \\
\hline \multirow{2}{*}{ Abril } & Hembra & 9 & 89,50 & 12,55 \\
\hline & Macho & 11 & 67,10 & 5,27 \\
\hline \multirow{2}{*}{ Mayo } & Hembra & 8 & 125,33 & 7,97 \\
\hline & Macho & 1 & 128,84 & 3,17 \\
\hline \multirow{2}{*}{ Junio } & Hembra & 0 & SD & SD \\
\hline & Macho & 0 & SD & SD \\
\hline \multirow{2}{*}{ Julio } & Hembra & 5 & 64,60 & 5,30 \\
\hline & Macho & 3 & 75,50 & 1,55 \\
\hline \multirow{2}{*}{ Agosto } & Hembra & 4 & 40,78 & 3,70 \\
\hline & Macho & 1 & 36,90 & 2,59 \\
\hline \multirow{2}{*}{ Septiembre } & Hembra & 6 & 119,07 & 10,74 \\
\hline & Macho & 1 & 106,00 & 2,16 \\
\hline
\end{tabular}

$\mathrm{SD}=\operatorname{Sin}$ dato 


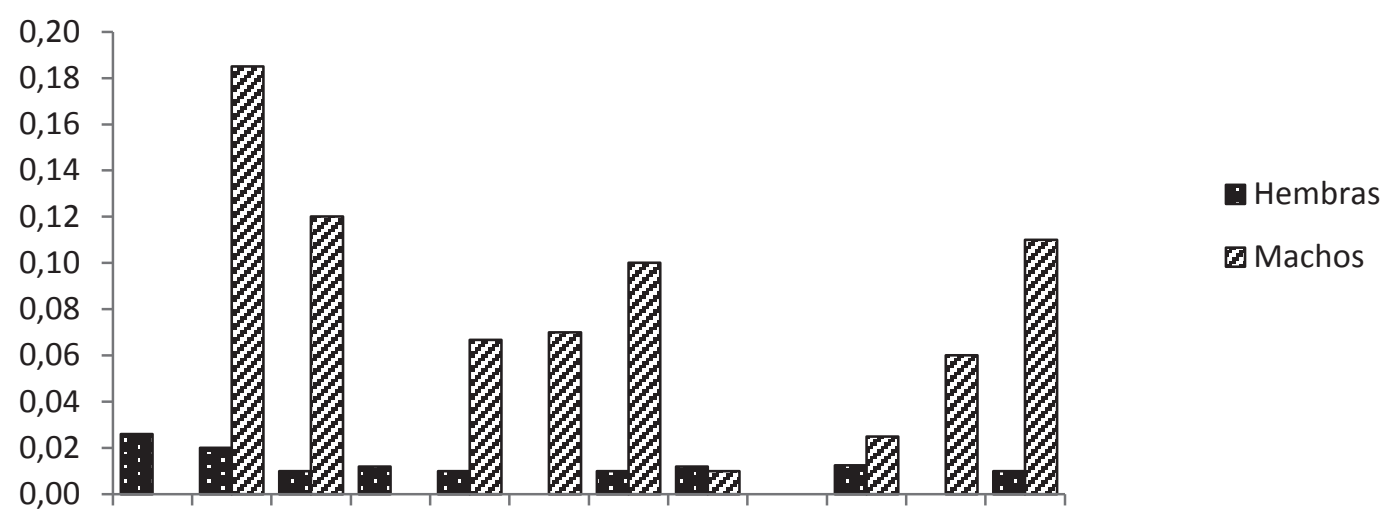

Oct. Nov. Dic. Ene. Feb. Mar. Abr. May. Jun. Jul. Ago. Sep.

Figura 1. Variación de los niveles plasmáticos de testosterona $(\mathrm{ng} / \mathrm{mL})$ en Eremophilus mutisii, capturados en el municipio de Suesca (Cundinamarca), a lo largo de un ciclo hidrológico. Las barras ilustran la media ( $\mathrm{n}=$ entre X y "Y".

Por otro lado, el hecho que se presenten varios picos con posterior descenso sería un indicativo que, a pesar de existir una época de mayor actividad reproductiva, la especie no tiene una única estación de desove en el año y que se podría reproducir varias veces durante el ciclo anual, tal como lo comprobaron Argungu et al. (2015), en Clarias batrachus, que al igual que el capitán de la sabana, es un silúrido de migraciones cortas.

Con relación a los niveles plasmáticos de estradiol (Figura 2 ), en las hembras oscilaron entre $0,04 \mathrm{ng} / \mathrm{mL}$, en julio y 0,3 , en marzo, presentado niveles elevados $(>0,2 \mathrm{ng} / \mathrm{mL})$ en noviembre, diciembre y enero, con pico en diciembre $(0,26 \mathrm{ng} /$ $\mathrm{mL}$ ). En los machos, solo fue posible determinar E2 en noviembre, diciembre, marzo, abril y mayo, puesto que durante febrero, junio, agosto y septiembre los valores fueron indetectable y, en los meses restantes, no se logró la captura de individuos machos. El valor máximo obtenido para ellos fue de $0,14 \mathrm{ng} / \mathrm{mL}$, en noviembre y, el mínimo, de 0,01, en marzo y abril.

La dinámica observada en el E2, a lo largo del año, permite corroborar lo ya expuesto, en el sentido de que la especie, probablemente, se reproduce varias veces al año, pues evidencia varios picos de esteroides durante el ciclo hidrológico. Tal situación sería muy importante si se quieren implementar programas de piscicultura, pues uno de los principales cuellos de botella en la producción es la estacionalidad reproductiva de las especies. En especial, tal dinámica se expresó en las hembras, lo cual, puede significar que la época de

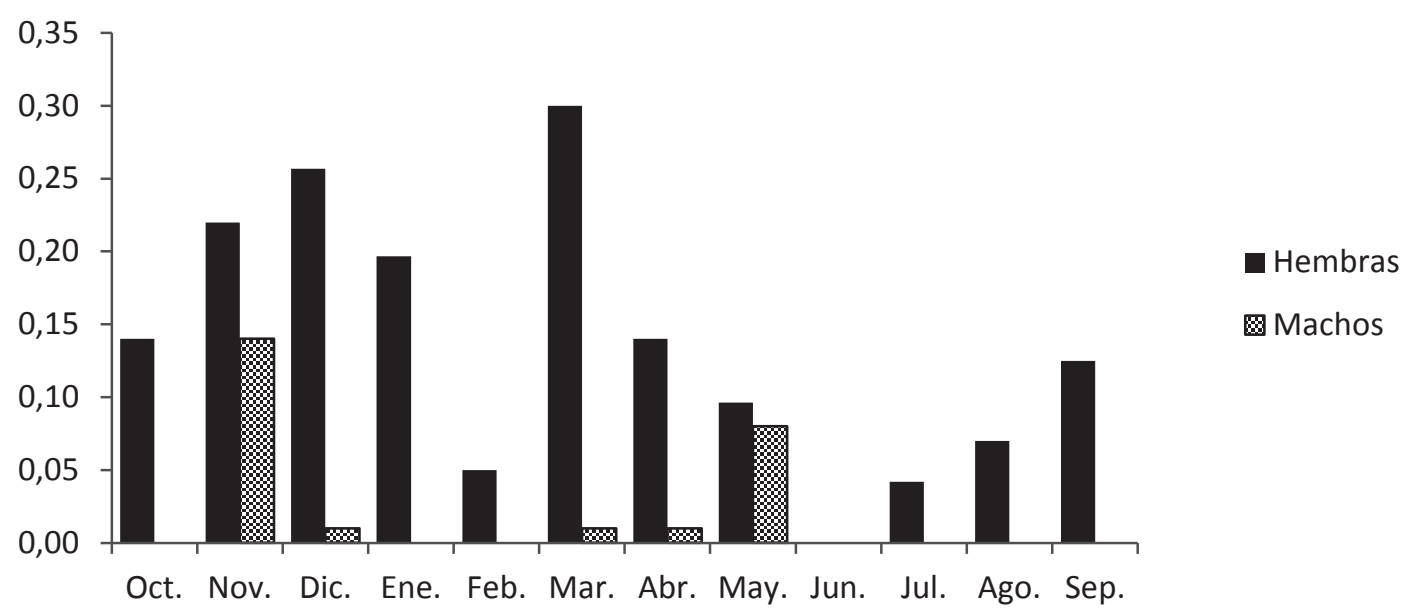

Figura 2. Valores medios de estradiol (ng/mL) de Eremophilus mutisii, capturados en el municipio de Suesca (Cundinamarca). 
desove estaría íntimamente relacionada con el nivel de estradiol circulante en plasma, resultado similar al encontrado por Harmin et al. (1995), en Pleuronectes americanus, especie que presentó una dinámica parecida, con aumentos y descensos del nivel de E2, a lo largo del ciclo reproductivo, tal como también fue reportado por Shabana et al. (2013), en Argyrosomus regius y, por Argungu et al. (2015), en Clarias batrachus.

Los resultados encontrados para ambos esteroides podrían ser explicados basándose en el hecho de que la especie no realiza grandes migraciones y su ciclo anual incluye varios periodos de reproducción, los cuales, estarían relacionados con los niveles plasmáticos de estradiol y de testosterona. En especies migratorias, con una única reproducción por ciclo, la dinámica esteroidal es diferente, presentando un solo pico durante todo el ciclo, que estaría asociado con la época previa al desove. Tales aspectos son consistentes con los hallados por Lokman et al. (1998), en anguilas migratorias y no migratorias. Estos autores explican el comportamiento de los esteroides según si la especie migra o no, concluyendo que, en las que migran, existe un único pico (muy alto) de ambos esteroides, al ser comparados con las especies que no migran, cuyos valores siempre son menores (Lokman \& Young, 1998).

Por otro lado, al relacionar los niveles plasmáticos de esteroides con el IGS, se puede entender más claramente la dinámica esteroidal con el grado de maduración de los animales, pudiéndose observar que, en las hembras, los picos de E2 coinciden con los valores más altos de IGS, mientras que la $T$ mantiene su nivel bajo, independientemente de dicho índice (Figura 3). En los machos (Figura 4), la relación entre T e IGS también es coincidente, aunque no de manera tan evidente como en las hembras y no se observa una tendencia clara entre la relación de E2 e IGS. De hecho, los datos obtenidos no serían suficientes para determinar tal relación, de manera concluyente, en los machos; no obstante, los resultados son coincidentes con los de especies de ambientes lénticos o que no realizan migraciones largas, como los obtenidos por Taghizadeh et al. (2013), en Cyprinus carpio y por otros autores, en especies cuyo desarrollo gonadal y dinámica esteroidal obedece a otros parámetros medioambientales diferentes a la migración (Cornish, 1998; Rahman et al. 2000; Norberg et al. 2004).

La dinámica esteroidal encontrada y los valores de IGS permiten suponer que, a pesar que el desarrollo gonadal tiene relación directa con el nivel de esteroides sexuales (Harmin et al. 1995), la especie no sigue un patrón estacional estricto, tal como lo reportan Adebiyi et al. (2013), en Hemibagrus nemurus y Argungu et al. (2015), en Clarias batrachus.

Con base en los resultados encontrados, se podría decir que el perfil de esteroides sexuales de Eremophilus mutisii guarda relación directa con el grado de maduración, en términos de IGS de los animales y que, al parecer, la especie se reproduce varias veces al año. Asimismo, se podría concluir que el conocimiento de la dinámica de los esteroides sexuales y su relación con el IGS pueden ser buenas herramientas para el entendimiento del ciclo reproductivo de la especie, con miras a su reproducción en cautiverio, tal como lo ha

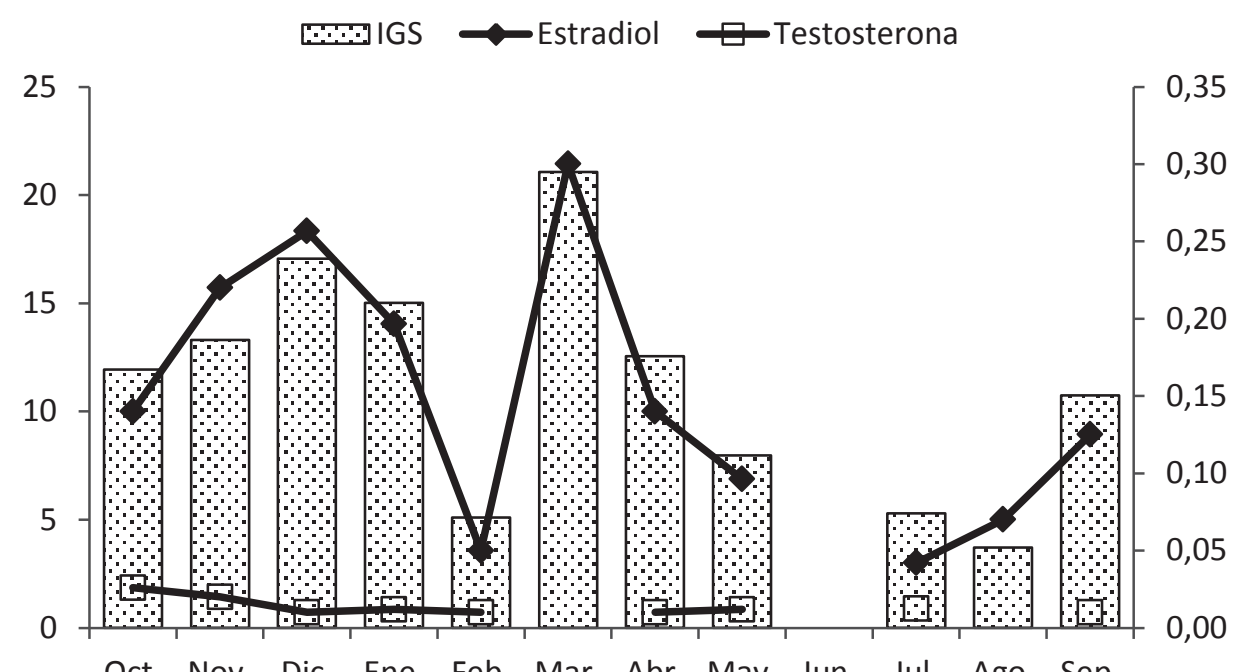

Figura 3. Variación de los índices gonadosomáticos (IGS) (\%) y de los niveles plasmáticos de estradiol (ng/mL) y testosterona $(\mathrm{ng} / \mathrm{mL})$, en hembras de Eremophilus mutisii, capturadas en el municipio de Suesca (Cundinamarca), a lo largo de un ciclo hidrológico. La figura ilustra la media $\pm \mathrm{DE}$. 


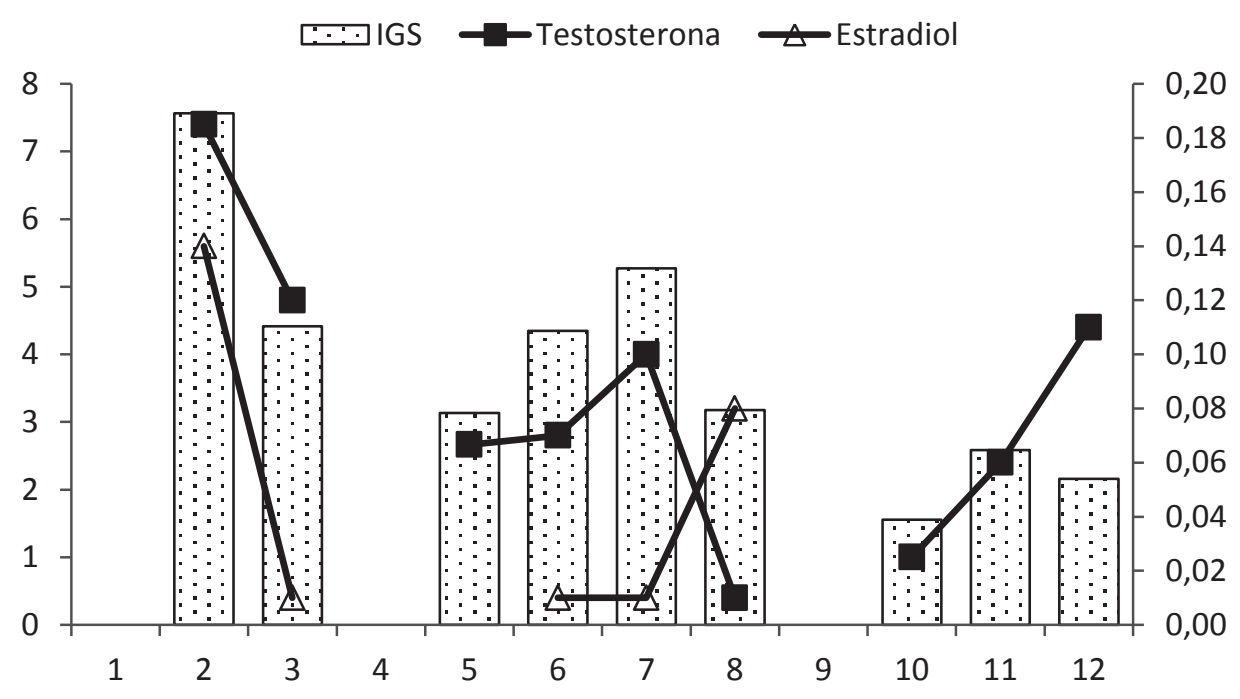

Figura 4. Valores medios de IGS (\%), testosterona (ng/mL) y estradiol $\mathrm{ng} / \mathrm{mL}$ ) en machos de Eremophilus mutisii, capturados en el municipio de Suesca (Cundinamarca).

sido para diferentes especies de peces, como Clarias macrocephalus (Tan-Fermin et al. 1997), Labeo rohita (Dasgupta et al. 2009), Arapaima gigas (Monteiro et al. 2010), Anguilla anguilla (Burgerhout et al. 2016), entre otras; sin embargo, futuras investigaciones, que incluyan edad y talla de maduración, áreas de desoves, proporción sexual, entre otros, tienen que llevarse a cabo, para continuar entendiendo la biología reproductiva de la especie.

Agradecimientos. Al señor Pablo Guáqueta, pescador del Municipio de Suesca, por su colaboración en la obtención de los ejemplares. Financiación. Este estudio fue financiado por la Vicerrectoría de Investigaciones de la Universidad de Ciencias Aplicadas y Ambientales U.D.C.A y por el Laboratorio de Fisiología de Peces de la Universidad Nacional de Colombia. Conflictos de intereses. El manuscrito fue preparado y revisado con la participación de todos los autores, quienes declaran que no existe conflicto de intereses que ponga en riesgo la validez de los resultados presentados.

\section{BIBLIOGRAFÍA}

1. ADEBIYI, F.; SIRAJ, S.; HARMIN, S.; CHRISTIANUS, A. 2013. Plasma sex steroid hormonal profile and gonad histology during the annual reproductive cycle of river catfish Hemibagrus nemurus (Valenciennes, 1840) in captivity. Fish Physiol. Biochem. 39:547557.

2. ARGUNGU, L.; CHRISTIANUS, A.; AMIN, M.; DAUD, S.; SIRAJ, S. 2015. Annual dynamics of the plasma sex steroid hormones of the Malaysian Walking Catfish
Clarias batrachus (Linnaeus 1758). J. Fish. Aquat. Sci. 10:24-34.

3. BURGERHOUT, E.; MINEGISHI, Y.; BRITTIJN, S.; DE WIJZE, L.; HENKEL, C.; JANSEN, H.; SPAINK, H.; DIRKS, R.; VAN DEN THILLART, G. 2016. Changes in ovarian gene expression profiles and plasma hormone levels in maturing European eel (Anguilla anguilla); Biomarkers for broodstock selection. General Comparat. Endocrinol. 225:185-196.

4. CORNISH, D. 1998. Seasonal steroid hormone profiles in plasma and gonads of the tilapia, Oreochromis mossambicus. Water SA. 24(3):257-264.

5. DASGUPTA, S.; SARKAR, S.; SARANGI, N.; BHATTACHARYA, S. 2009. Variation in spawning responses, egg and larvae productions from induced rohu (Labeo rohita) during pre-monsoon and monsoon seasons: Relationship with hormonal changes and oocyte responsiveness during final maturation. Aquaculture. 290:320-326.

6. GONZÁLEZ, J.; ROSADO, R. 2005. Reproducción inducida con hormonas en Eremophilus mutisii Humboldt, 1805 (Pisces: Trichomycteridae) en Guasca Cundinamarca, Colombia. Revista de Investigaciones Universidad de La Salle. 5(2):233-240.

7. GONZÁLEZ, J.; ROSADO, R. 2010. Estado del conocimiento sobre peces nativos del altiplano cundiboyacense: revisión. Rev. Cien. Anim. 3:69-79. 
8. HARMIN, S.; CRIM, W.; WIEGAND, M. 1995. Plasma sex steroid profiles and the seasonal reproductive cycle in male and female winter flounder, Pleuronectes americanus. Marine Biology. 121:601-610.

9. LOKMAN, P.; YOUNG G. 1998. Gonad histology and plasma steroid profiles in wild New Zealand freshwater eels (Anguilla dieffenbachii and A. australis) before and at the onset of the natural spawning migration. II. Males. Fish Physiology and Biochemistry. 19:339-347.

10. LOKMAN, P.; VERMEULEN, G.; LAMBERT, J.; YOUNG, G. 1998. Gonad histology and plasma steroid profiles in wild New Zealand freshwater eels (Anguilla dieffenbachii and $A$. australis) before and at the onset of the natural spawning migration. I. Females. Fish Physiology and Biochemistry. 19:325-338.

11. MALDONADO, O.; VARI, R.; USMA, S. 2008. Checklist of the freshwater fish of Colombia. Rev. Biota Colombia. 9(2):44-60.

12. MOJICA, I.; CASTELLANOS, C.; USMA, S.; ÁLVAREZ, R. (Eds). 2002. "Libro rojo de peces dulceacuícolas de Colombia". Serie Libros Rojos de Especies Amenazadas de Colombia. Instituto de Ciencias Naturales Universidad Nacional de Colombia, Ministerio del Medio Ambiente, Bogotá, Colombia.

13. MOJICA, I.; USMA, S.; ÁLVAREZ, R.; LASSO, C. (Eds). 2012. "Libro rojo de peces dulceacuícolas de Colombia". Serie Libros Rojos de Especies Amenazadas de Colombia. Instituto de Investigación de Recursos Biológicos Alexander von Humboldt, Ministerio del Medio Ambiente, Bogotá, Colombia.

14. MONTEIRO, L.; SOARES, M.; CATANHO, M.; HONCZARYK, A. 2010. Aspectos reprodutivos e perfil hormonal dos esteróides sexuais do pirarucu, Arapaima gigas (Schinz, 1822), em condições de cativeiro. Acta Amazônica. 40 (3):435-450.

15. NORBERG, B.; BROWN, C.; HALLDORSSON, O.; STENSLANDD, K.; BJÖRNSSON, B. 2004. Photoperiod regulates the timing of sexual maturation, spawning, sex steroid and thyroid hormone profiles in the Atlantic cod (Gadus morhua). Aquaculture. 229:451-467.

16. RAHMAN, S.; TAKEMURA, A.; TAKANO, K. 2000. Correlation between plasma steroid hormones and vitellogenin profiles and lunar periodicity in the female golden rabbitfish, Siganus guttatus (Bloch). Comparative Biochemistry and Physiology Part B. 127:113122.

17. RODRÍGUEZ, A.; ROSADO, R. 1993. Ensayos de reproducción inducida en capitán de la sabana Eremophilus mutisii, Humboldt, 1805. Boletín Red Regional de Acuicultura CIID-Colciencias. 7(3):10-13.

18. ROSADO, R.; GONZÁLEZ, J. 2007. Compilación bibliográfica y análisis del estado actual de la investigación sobre capitán de la sabana, Eremophilus mutisii. Dahlia-Rev. Asoc. Colomb. Ictiol. 9:43-51.

19. SALCEDO, A.; DÍAZ, S.; GONZÁLEZ, J.; RODRÍGUEZ, A.; VARONA, M. 2012. Exposición a plaguicidas en los habitantes de la ribera del río Bogotá (Suesca) y en el pez capitán. Rev. Cienc. Salud. 10(Especial):29-41.

20. SCHIAVONE, R.; ZILLI, L.; STORELLI, C.; VILELLA, S. 2012. Changes in hormonal profile, gonads and sperm quality of Argyrosomus regius (Pisces, Scianidae) during the first sexual differentiation and maturation. Theriogenology. 77:888-898.

21. SHABANA, N.; EL RAHMAN, S.; AL ABSAWY, M.; ASSEM, S. 2013. Reproductive biology of Argyrosomus regius (Asso, 1801) inhabiting the south eastern Mediterranean Sea, Egypt. Egyptian Journal of Aquatic Research. 38:147-156.

22. SISNEROS, J.; FORLANO, P.; KNAPP, R.; BASS, A. 2004. Seasonal variation of steroid hormone levels in an intertidal-nesting fish, the vocal plainfin midshipman. Gen. Comp. Endocrinology. 136:101-116.

23. TAGHIZADEH, V.; IMANPOOR, M.; MEHDINEJAD, N. 2013. Study the seasonal steroid hormones of common carp in Caspian Sea, Iran. Springer Plus Journal. 2:193-196.

24. TAN-FERMIN, J.; IJIRI, S.; UEDA, H.; ADACHI, S.; YAMAUCHI, K. 1997. Ovarian development and serum steroid hormone profiles in hatchery-bred female catfish Clarias macrocephalus (Gunther) during an annual reproductive cycle. Fisheries Science. 63(6):867-872.

25. VALDERRAMA, M.; MORALES-BETANCOURT, M.; HERNÁNDEZ, S. 2011. Eremophilus mutisii. En: Lasso, C.A.E.; Agudelo Cordoba, L.F., Jimenez-Segura, H.; Ramirez-Gil, M.; Morales-Betancourt, R.E.; Ajiaco- 
Martínez, F.; de Paula Gutierrez, F.; Usma Oviedo, Recibido: Noviembre 30 de 2016 J.S.; Muñoz Torres, S.E.; Sanabria Ochoa, A.L. (Eds). Aceptado: Abril 30 de 2017 Catálogo de los recursos pesqueros continentales de Colombia. Serie Editorial Recursos Hidrobiológicos y Pesqueros Continentales de Colombia. Instituto de Investigación de Recursos Biológicos Alexander von Humboldt. Bogotá, D.C., Colombia, 715p.

\section{Cómo citar:}

Landines P., M.A.; Prieto M., C.A.; Rodríguez V., L.; Rosado P., R. 2017. Perfil de esteroides sexuales del capitán de la sabana (Eremophilus mutisii) durante un ciclo hidrológico completo. Rev. U.D.C.A Act. \& Div. Cient. 20(1): 43-50. 